\title{
Spectral-spatial structure of the high-power laser diodes radiation during their operation
}

\author{
Vladimir Bliznyuk ${ }^{1, *}$, Olga Koval $^{1}$, Vasiliy Parshin $^{1}$, Alexey Rzhanov $^{2}$, Alexander Tarasov $^{1}$, \\ and Vladislav Grigoriev ${ }^{1}$
}

${ }^{1}$ National Research University "Moscow Power Engineering Institute", 111250 Moscow, Russia

${ }^{2}$ Lomonosov Moscow State University, Faculty of Physics, 119991 Moscow, Russia

\begin{abstract}
The emission spectra of high-power laser diodes in the process of exploitation at different pump currents and the relationship of these spectra with the spatial structure of the field are considered. The field in laser diodes with a wide contact splits into several independent channels corresponding to the components of the radiation frequency spectrum. It is established that the number of channels and the corresponding number of spectral components of laser diode radiation increases during operation.
\end{abstract}

Powerful semiconductor laser diodes (LD) with quantum-well hetero-structures are widely used in science, engineering, medicine and other fields. Such lasers are usually referred to as LD with a continuous generation power from several hundred milliwatts to almost $20 \mathrm{~W}$ [1-5]. The devices degrade due to the heating of the active region and the waveguide, the gradual diffusion of atoms and stress relief at the contact points of semiconductor layers. Studies show that the lifetime of 0.5-2.0 W LD does not exceed 5000 hours [6]. There are several methods for predicting the lifetime of powerful LD [5]. However, the use of these techniques involves the expenditure of a significant resource of lasers, the use of expensive instrumentation, high time cost and the need to interrupt the work of the LD for measurements. In this regard, the task of studying the effect of degradation of the laser structure on the radiation of high-power LD's, and predicting their service life not only in a short time, but also at the initial stage of their operation becomes urgent.

In the testing process of LDs, which were manufactured in 2012, we observed the transformation of their radiation spectrum [7, 8]. This transformation has manifested itself in the change from three clearly expressed spectral lines corresponding to three spatial channels of LD generation to 4-5 spectral lines corresponding to 4-5 channels.

We associate changes in the spectrum of the device with spatial variations, which relate to the coefficient of nonlinear refraction of the quantum well semiconductor and the coherence length of the LD radiation. Our calculations [7] showed that the number of generation channels is directly related to the value of the coherence length of each channel (it is assumed that these values for the channels are close to each other). The smaller number of generation channels corresponds to the higher degree of coherence. So, the

\footnotetext{
* Corresponding author: 4059541@ mail.ru
} 
decrease of the coherence length $L_{c o h}$ increases the number $N_{c h}$ of non-phase-locked laser space channels:

$$
N_{c h} \approx n W \sqrt{\frac{2 \pi}{\lambda L_{c o h}}},
$$

where $n$ is the average effective refractive index of the fundamental transverse mode of the laser waveguide, $W$ is the width of the active region of $\operatorname{LD}(100 \mu \mathrm{m}), \lambda$ is the average wavelength of radiation in vacuum $(970 \mathrm{~nm})$. The calculation according to the formula (1) gives the value of $L_{c o h}$ about $4 \mathrm{sm}$ at two channels of radiation, which is quite consistent with the results of experiments on the measurement of the $L_{c o h}$ value in LD [9].

Currently, we are studying the spectral characteristics of LD manufactured in 2017. They have the same design as the LD from the previous batch. In the first hours of their operation in their radiation were recorded two lines of generation. In accordance with (1), this allows us to conclude that the coherence length of such LDs is greater than that of the LD from the previous batch, in which the diodes had three pronounced peaks in the radiation spectrum at the beginning of operation. As developments 200 hours of operation in the spectrum party LD 2017 release occur three rather than two visible lines are generated. The separation of these lines from the total spectral curve with the determination of the peak frequency in each line $v_{01}<v_{02}<v_{03}$ is an independent problem. The algorithm for solving these lines is developed in this work.

The feature of the generation lines analyzis at frequencies $v_{01}, v_{02}, v_{03}$ is that is provides at the wide range of pump current values. At the lower current value the peak frequency $v_{02}$ radiation prevails. At the higher pump current value the peak frequency $v_{01}$ prevails. Each of the three lines can be characterized by its quality factor (Q-factor), which is determined by the ratio of the peak frequency to the width of the corresponding radiation line. From the analysis of the three components of the radiation spectrum, it is found that the Q-factor of the line with the peak frequency $v_{01}$ is greater than the Q-factor of the lines with the peak frequencies $v_{02}$ and $v_{03}$. With an increase in the pump current, there a competition of transitions with frequencies $v_{01}$ and $v_{02}$ takes plase. Ultimately, the energy passes more and more into the laser transition with frequency $v_{01}$.

In our opinion, each line corresponds to a spatial generation channel in the active region. Analysis of the shape of these lines shows that they usually differ from the Gaussian curves with central frequencies $v_{01}, v_{02}, v_{03}$. This difference increases with the pump current increase. According to the technique described in [10], the degree of this difference can surve the prediction of LD lifetime.

\section{References}

1. A.P. Bogatov, A.E. Drakin, A.A. Stratonnikov et al., QE 30, 401 (2000)

2. S.O. Slipchenko, A.A. Bondarev, D.A. Vinokurov et al., FTP, 43, 119 (2009)

3. S.O. Slipchenko, D.A. Vinokurov, N.A. Pikhtin et al., FTP, 38, 1477 (2004)

4. A.V. Lutetsky, N.A. Pikhtin, N.V. Fetisova et al., FTP, 43, 1646 (2009)

5. A.E. Zhukov, Physics and technology of semiconductor nanostructures (Elmore, St. Petersburg, 2007)

6. L.E. Vorobjev, A.N. Sofronov, D.A. Firsov et al., Photonics, 31, 20 (2012)

7. V.V. Bliznyuk, O.I. Koval, A.G. Rzhanov et al., Izv. RAN Ser. Fiz., 79, 12 (2015)

8. V.V. Bliznyuk, O.I. Koval, V.A. Parshin et al., Bull. MSU. Phys., 6, 1860501 (2018)

9. S.P. Vorobjev, http://www.holography.ru/files/holmich.htm (2018)

10. V.V. Bliznyuk, O.I. Koval, V.A. Parshin et al., Izv. RAN Ser. Fiz., 82, 11 (2018) 